\title{
Prevalência de depressão em mulheres idosas assistidas na atenção básica
}

\author{
Prevalence of depression in elderly women assisted in basic attention
}

\author{
Hellen Cristina Almeida Abreu de Lara ${ }^{a}$ \\ Orcid: https://orcid.org/0000-0001-5845-3895 \\ Cristiana Alves de Melob \\ Orcid: https://orcid.org/0000-0002-2219-5633 \\ Evening da Cruz Silva ${ }^{c}$ \\ Orcid: https://orcid.org/0000-0002-8615-6422
}

\author{
Izabel Aparecida da Silva ${ }^{d}$ \\ Orcid: https://orcid.org/0000-0003-4883-4533 \\ Jefferson Souza de Oliveira ${ }^{\mathrm{e}}$ \\ Orcid: https://orcid.org/0000-0001-8581-561X \\ Franciel Souza Santana ${ }^{f}$ \\ Orcid: https://orcid.org/0000-0003-4450-1790
}

\begin{abstract}
Resumo
Introdução: A depressão em idosas apresenta diversos fatores de risco como, precárias condições socioeconômicas e condições de saúde. Ela é frequentemente ignorada, pois, em geral, os profissionais de saúde veem os sintomas depressivos como manifestações normais decorrentes do processo do envelhecimento. Objetivo: Analisar a prevalência de sintomas de depressão em idosas cadastrados em uma Unidade Básica de Saúde do município de Várzea Grande, Mato Grosso. Método: Estudo transversal, realizado com pessoas de 60 anos ou mais, sexo feminino, com uso de um questionário com dados sociodemográficos, condições de saúde e a Escala de Depressão Geriátrica. A análise de dados foi feita por meio de medidas de frequência simples, absoluta e calculadas razões de prevalências brutas e ajustadas com intervalo de confiança de 95\% no Programa Epiinfo 7.2. Resultados: A prevalência de depressão foi de $18,00 \%$. O uso de medicamentos, grau de dependência, morte de familiares e alguém na família com depressão foram associados à depressão em idosas na comunidade. Conclusão: Dessa forma, é necessário a detecção precoce dos sintomas depressivos em idosas e fatores associados, e considerar o sexo feminino como algo importe pois auxilia os profissionais da atenção básica a compreender a realidade e ainda minimizar os danos para a saúde e qualidade de vida dos idosos.
\end{abstract}

Palavras-chave: depressão; saúde do idoso; atenção primária à saúde.

\begin{abstract}
Introduction: Depression in the elderly has several risk factors such as poor socioeconomic conditions and health conditions. It is often overlooked because health professionals often see depressive symptoms as normal manifestations of the aging process. Objective: To analyze the prevalence of symptoms of depression in elderly women enrolled in a Basic Health Unit in the municipality of Varzea Grande, Mato Grosso. Method: Cross-sectional study, conducted with women aged 60 years or older, using a questionnaire with sociodemographic data, health conditions and the Geriatric Depression Scale. Data analysis was performed using simple, absolute frequency measurements and calculated crude and adjusted prevalence ratios with a
\end{abstract}

\footnotetext{
${ }^{a}$ Mestre em Enfermagem. Centro Universitário de Várzea Grande (UNIVAG) pelo Programa de Pós-Graduação de Enfermagem da Universidade Federal de Mato Grosso - UFMT, E-mail: afa nany@yahoo.com.br

b Graduação em Enfermagem. Centro Universitário de Várzea Grande (UNIVAG), Brasil, E-mail: cristianaalvesmelo@hotmail.com ${ }^{\mathrm{c}}$ Graduação em Enfermagem. Centro Universitário de Várzea Grande (UNIVAG), Brasil, E-mail: eveningbebe2@hotmail.com

${ }^{d}$ Graduação em Enfermagem. Centro Universitário de Várzea Grande (UNIVAG), Brasil, E-mail: izza_bl@hotmail.com

e Acadêmico de Enfermagem pelo Centro Universitário de Várzea Grande (UNIVAG), Brasil. E-mail: jefferson.15souza.js@gmail.com

${ }^{\mathrm{f}}$ Acadêmico de Enfermagem. Centro Universitário de Várzea Grande (UNIVAG), Brasil, E-mail: francielsantana1010@hotmail.com
} 
95\% confidence interval in the Epiinfo 7.2 Program. Results: The prevalence of depression was $18.00 \%$. Medication use, degree of dependence, death of family members, and someone in the family depression were associated with depression in older women in the community. Conclusion: Thus, early detection of depressive symptoms in elderly women and associated factors is necessary and considering female gender as important because it helps primary care professionals to understand reality and minimize the damage to health and quality of life. of the elderly.

Keywords: depression; health of the elderly; primary health care.

\section{Introdução}

No atual século, vem ocorrendo de forma contínua o aumento do número de idosos mundialmente, principalmente em países em desenvolvimento. No Brasil, cogita-se que até 2020 os idosos corresponderão a $15 \%$ da população brasileira. Já em 2025, projeta que a população idosa deverá ter aumento de até 15 vezes, enquanto a população total deverá ter crescido apenas 5 vezes, colocando o Brasil em sexto lugar no ranking dos países $^{1,2}$.

Assim, o processo de envelhecer provoca mudanças tanto nas funções quanto na estrutura do corpo e o torna mais propenso a uma série de fatores nocivos à saúde, esses podem ser tanto internos como externos $^{3}$. Dentre esses fatores, estão as doenças crônicas, as mais comuns são Hipertensão Arterial Sistêmica (HAS) e o Diabetes mellitus (DM), sendo consideradas como os principais fatores de risco para o desenvolvimento de complicações renais, doenças cardíacas e cerebrovasculares. Tendo como consequências os altos custos médicos e socioeconômicos, decorrentes principalmente das complicações que as acompanham ${ }^{4}$.

Além disso, existem outras doenças crônicas que acometem os idosos, porém em menor proporção. Destaca-se a prevalência de doenças neurológicodegenerativas e as tendências à depressão. A depressão é a doença psiquiátrica mais comum entre os idosos. Ela afeta sua qualidade de vida, aumentando a carga econômica por seus custos diretos e indiretos e pode levar a tendências suicidas.
E tornou-se um problema de saúde pública, devido à elevada frequência com que ocorre $^{5,6}$.

Um Relatório da Organização Mundial de Saúde (OMS) aponta que o número de casos de depressão aumentou 18\% entre 2005 e 2015: são 322 milhões de pessoas em todo o mundo, a maioria mulheres. No Brasil, a prevalência de depressão entre as pessoas idosas varia de $4,7 \%$ a $36,8 \%$. As mulheres apresentam prevalências maiores que os homens na proporção de $2: 1^{7}$. O estudo de Gonçalves ${ }^{8}$ mostrou que a prevalência de depressão é mais significativa entre as mulheres que vivem em áreas urbanas, com níveis educacionais mais baixos e com condições crônicas. Nos estudos desenvolvidos no Brasil, o que aponta lacunas na assistência e nas políticas públicas e pouco investimento em pesquisas nesse âmbito. A depressão se apresenta como uma das principais causas de incapacitação no mundo, limitando o funcionamento físico, pessoal e social ${ }^{9,10}$.

Outrossim, a mulher idosa torna-se mais vulnerável ao risco de depressão, pois é nessa fase que a mulher se depara com várias transformações, que vão desde o climatério a mudanças relacionadas à senescência do envelhecimento e à presença de doenças crônicas, diminuindo a vontade de viver e assim levando a desenvolver sintomas depressivos ${ }^{11}$.

Dessa forma, a presença de depressão entre essa população feminina tem impacto negativo em sua vida. Assim, torna-se necessário que a Atenção Básica seja porta de entrada dessas mulheres, para ações de prevenção, promoção e tratamento. Torna-se, fundamental a construção de um projeto terapêutico 
singular (PTS), a partir do acolhimento e da avaliação, incluindo diferentes estratégias que possam atender às necessidades dessas mulheres ${ }^{9}$. Dessa forma, este trabalho visa analisar a prevalência de depressão em idosas assistidas pela Estratégia de Saúde da Família, Várzea Grande, Mato Grosso.

\section{Metodologia}

Estudo descritivo de corte transversal realizado a partir de uma amostra do projeto "A prevalência de depressão em idosos assistidos nas Unidades de Saúde da Família em Várzea Grande, Mato Grosso", realizada por Abreu $^{12}$. Sendo a amostra não probabilística por conveniência, pois considerou o cenário escolhido devido apresentar a maior quantidade de idosas e em situação de vulnerabilidade.

Dessa maneira, adotou-se como critério de inclusão todas as pessoas de 60 anos ou mais, do sexo feminino que são cadastradas e acompanhadas pela Unidade de Saúde da Família (USF) Gonçalo Pinto Godoy, em Várzea Grande. Foram excluídas dos estudos, as idosas que não estavam em domicílio, internadas ou falecidas durante a pesquisa, e aquelas que apresentaram dificuldades cognitivas e mentais que impediam de responder o questionário, sendo avaliado pela aplicação do Mini Exame do Estado Mental (MEEM).

A coleta de dados ocorreu entre os dias de 11 fevereiro a 07 de junho de 2019 . Utilizando o questionário fechado, com dados sociodemográficos, condições de saúde, Instrumento de Avaliação do Grau de Dependência para as Atividades Instrumentais da Vida Diária (AIVD) ${ }^{13} \mathrm{e}$ Instrumento de Avaliação do Grau de Dependência para as Atividades da Vida Diária (AVD) ${ }^{14}$ e a Escala Abreviada de Depressão Geriátrica (EDGA) $)^{15}$.

Para a presente pesquisa foi feito reunião com o responsável técnico da Unidade de USF e montada uma escala de visita domiciliar aos idosos, com o acompanhamento dos Agentes
Comunitários de Saúde (ACS). Assim, durante a visita domiciliar, as idosas eram informadas sobre a pesquisa, conforme a resolução 466/2012, após isso era solicitada a autorização da pesquisa por meio da assinatura do Termo de Consentimento Livre e Esclarecido (TCLE) e por último era aplicado o questionário da pesquisa.

Os questionários foram codificados e digitados no programa Epiinfo versão 7.2. Na análise descritiva, os dados foram realizados por meio de tabelas de frequência, sendo representadas por frequência absoluta (n) e relativa (\%). $\mathrm{Na}$ análise bivariada foram calculadas as Razões de Prevalência (RP) como medida de associação. Para o cálculo da significância estatística da associação valores menores de 5\%, sendo $(\mathrm{p}=$ valor $<0,05)$, utilizou-se o teste de Quiquadrado com intervalo de $95 \%$ de confiança pelo método de Mantel-Haenszel (IC 95\%), ou teste exato de Fisher quando indicado.

A análise estatística dos dados foi utilizada como variável-resposta à medida da Escala de Depressão Geriátrica, dicotomizada, adotando-se como ponto de corte para a avaliação de sintoma depressivo o valor seis. Idosos com valores abaixo de seis, considerados normais, enquanto aqueles com valores acima ou igual a seis foram avaliados como portadores de sintomas depressivos. O projeto contou com a aprovação do Comitê de ética e Pesquisa do Centro Universitário de Várzea Grande (CEP/UNIVAG) n ${ }^{\circ}$ do parecer 2.977.581.

\section{Resultado}

Participaram do presente estudo 250 idosas. Dentre elas, 45 apresentaram sinais de depressão $(18,00 \%)$. Conforme a tabela 1, população com faixa etária de 60 a 69 anos $(66,00 \%)$, casada $(62,80 \%)$, analfabeta $(63,60 \%)$ e renda de 1 salário mínimo $\quad(68,80 \%)$, proveniente principalmente de aposentadoria e benefícios. Além disso, 45,20\% moram com o cônjuge somente. 
Tabela 1 - Distribuição das idosas segundo características sociodemográficas. Várzea Grande - MT, 2019.

\begin{tabular}{|c|c|c|}
\hline Variável & Frequência (n) & Porcentagem (\%) \\
\hline \multicolumn{3}{|l|}{ Faixa etária } \\
\hline $60-69$ anos & 165 & 66,00 \\
\hline $70-79$ anos & 74 & 29,60 \\
\hline 80 anos ou mais & 11 & 4,40 \\
\hline \multicolumn{3}{|l|}{ Estado conjugal } \\
\hline Casada/união estável & 157 & 62,80 \\
\hline Viúva & 62 & 24,80 \\
\hline Solteira & 26 & 10,40 \\
\hline Separada/ divorciada & 05 & 2,00 \\
\hline \multicolumn{3}{|l|}{ Escolaridade } \\
\hline Analfabeta & 159 & 63,60 \\
\hline Primeiro Grau & 86 & 34,40 \\
\hline Segundo Grau & 05 & 2,00 \\
\hline \multicolumn{3}{|l|}{ Renda } \\
\hline Não possui & 32 & 12,80 \\
\hline Menos de 1 SM & 48 & 19,20 \\
\hline $1 \mathrm{SM}$ & 152 & 60,80 \\
\hline $2 \mathrm{SM}$ & 17 & 6,80 \\
\hline $3 \mathrm{SM}$ & 01 & 0,40 \\
\hline \multicolumn{3}{|l|}{ Com quem mora } \\
\hline Sozinha & 41 & 16,40 \\
\hline Cônjuge & 113 & 45,20 \\
\hline Cônjuge e filhos & 46 & 18,40 \\
\hline Cônjuge, filhos, genro ou nora & 04 & 1,60 \\
\hline Somente com filhos & 33 & 13,20 \\
\hline Somente com os netos & 9 & 3,60 \\
\hline Não familiares & 1 & 0,40 \\
\hline Filho (s), genro ou nora, neto & 3 & 1,20 \\
\hline Total & 250 & 100,00 \\
\hline
\end{tabular}

Em relação às condições de saúde, $(79,44 \%)$ das idosas reportaram ter problemas de saúde e os mais frequentes foram hipertensão arterial $(62 \%)$ e diabetes de mellitus (34,40\%). Além disso, (77,22\%) das idosas fazem uso de algum de medicamento, sendo que $(65,3 \%)$ fazem uso de dois medicamentos e $(10,04 \%)$ apresentam problemas auditivos. Apenas (6\%) declaram saúde ótima e $(36,40 \%)$ referiram a saúde como boa. (Tabela 2).

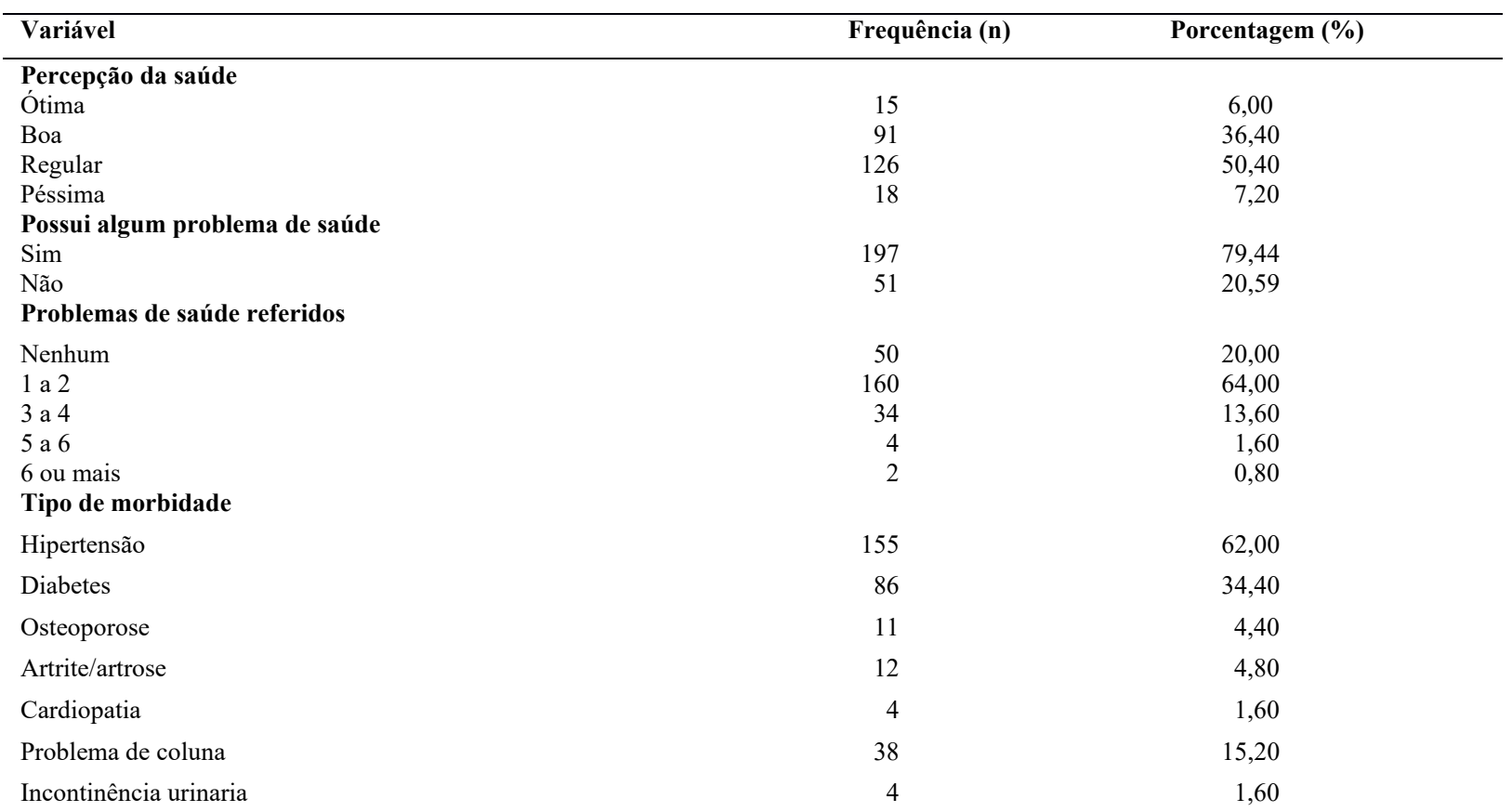




\begin{tabular}{lcc}
\hline Variável & Frequência (n) & Porcentagem (\%) \\
\hline Outra Qualquer & 29 & 11,60 \\
Uso de medicamentos & 183 & 77,22 \\
Sim & 54 & 22,78 \\
Não & & 10,04 \\
Condição de saúde & 25 & 16,53 \\
Audição & 41 & 10,53 \\
Marcha & 26 & 18,07 \\
Equilíbrio & 45 & 11,38 \\
Atividade física & 28 & 7,44 \\
Uso de Tabaco & 18 & 2,87 \\
Uso de Álcool & 07 & 20,73 \\
Uso de drogas & 51 & 42,34 \\
Familiares com depressão & 105 & 70,80 \\
Morte na família & & 29,20 \\
AIVD & 177 & 95,20 \\
Independente & 43 & 4,80 \\
Dependente & & \\
AVD & 238 & 82,00 \\
Independente & 12 & 18,00 \\
Dependente & & 100,00 \\
Escala de depressão geriátrica - GDS & 205 & \\
Menor ou igual a 5 (sem sintomatologia depressiva) & 45 & 250 \\
Maior que 5 (com sintomatologia depressiva) & & \\
\hline Total & &
\end{tabular}

Fonte: Dados da Pesquisa

$\mathrm{Na}$ análise bivariada, em relação à renda familiar da idosa até 01 saláriomínimo ( $\mathrm{RP}=1,94$; IC $95 \%=0,23-8,73 \mathrm{p}=$ $0,378)$, ser viúva $(\mathrm{RP}=1,30$; IC $95 \%=0,76-$ $2,21 ; \mathrm{p}=0,326)$ e em relação ao nível de escolaridade ser analfabeto $(\mathrm{RP}=10,94$; IC $95 \%=0,54-1,62 ; \mathrm{p}=0,832)$, no entanto não houve associação estatisticamente significativa.

Tabela 3 - Distribuição das idosas que apresentam sintomas de depressão segundo variáveis sociodemográficas. Várzea Grande, 2019.

\begin{tabular}{|c|c|c|c|c|c|c|}
\hline \multirow[t]{3}{*}{ Variáveis } & \multicolumn{4}{|c|}{ Depressão (EDG) } & \multirow{3}{*}{ RP (IC 95\%) } & \multirow{3}{*}{$P$ valor } \\
\hline & \multicolumn{2}{|c|}{ Maior que 5} & \multicolumn{2}{|c|}{ Menor ou igual a 5} & & \\
\hline & $\mathrm{N}$ & $\%$ & $\mathrm{~N}$ & $\%$ & & \\
\hline \multicolumn{7}{|l|}{ Faixa etária } \\
\hline Até 69 anos & 30 & 18,18 & 135 & 81,82 & & \\
\hline 70 anos ou mais & 15 & 17,65 & 70 & 82,35 & $0,97(0,55-1,70)$ & 0,917 \\
\hline \multicolumn{7}{|l|}{ Estado civil } \\
\hline Casada & 25 & 16,13 & 130 & 83,87 & & \\
\hline Divorciada/solteira/viúva & 20 & 21,05 & 75 & 78,95 & $1,30(0,76-2,21)$ & 0,326 \\
\hline \multicolumn{7}{|l|}{ Renda da idosa } \\
\hline Não possui/1SM & 43 & 18,61 & 188 & 81,39 & $1,94(0,43-8,73)$ & 0,378 \\
\hline Acima 1 SM & 2 & 10,53 & 17 & 89,47 & & \\
\hline \multicolumn{7}{|l|}{ Escolaridade } \\
\hline Analfabeta & 17 & 18,68 & 28 & 17,61 & $0,94(0,54-1,62)$ & 0,832 \\
\hline Primeiro e segundo Grau & 74 & 81,32 & 131 & 82,39 & & \\
\hline \multicolumn{7}{|l|}{ Com quem mora } \\
\hline Sozinha & 6 & 14,63 & 35 & 85,37 & $0,78(0,35-1.73)$ & 0,540 \\
\hline Com familiares & 39 & 18,66 & 170 & 81,34 & & \\
\hline
\end{tabular}

RP: Razão de Prevalência; IC 95\%: Intervalo de Confiança para a proporção de 95\%; *teste de Fisher

Fonte: Dados da Pesquisa

A Tabela 4 contempla a distribuição das idosas, as condições de saúde e as variáveis que apresentaram associações estatísticas com o desfecho foram a de fazer uso de medicamentos $(\mathrm{RP}=$ $0,34$; IC $95 \%=0,13-0,92 ; p=0,012)$, score de AIVD $(\mathrm{RP}=2,31 ; \mathrm{IC} 95 \%=1,38-3,88 ; \mathrm{p}=$ $0,001)$ e o grau de AVD (RP- 2,47 ; IC $95 \%=$ $1,19-5,12 ; \mathrm{p}=0,044)$, alguém na família com depressão $(\mathrm{RP}=3,70$; IC $95 \%=2,24$ $6,12 ; \mathrm{p}=<0,001)$ e morte na família $(\mathrm{RP}=1,72 ; \mathrm{IC} 95 \%=1,01-2,93 \mathrm{p}=0,031)$. 
Tabela 4 - Distribuicão das idosas que apresentam sintomas de depressão segundo condicões de saúde. Várzea Grande - MT, 2019.

\begin{tabular}{|c|c|c|c|c|c|c|}
\hline \multirow[t]{3}{*}{ Variáveis } & \multicolumn{4}{|c|}{ Depressão (EDG) } & \multirow[t]{3}{*}{ RP (IC 95\%) } & \multirow[t]{3}{*}{ p-valor } \\
\hline & \multicolumn{2}{|c|}{ Maior que 5} & \multicolumn{2}{|c|}{ Menor ou igual a 5} & & \\
\hline & $\mathrm{N}$ & $\%$ & $\mathrm{~N}$ & $\%$ & & \\
\hline \multicolumn{7}{|l|}{$\begin{array}{l}\text { Possui algum problema de } \\
\text { saúde }\end{array}$} \\
\hline Sim & 6 & 11,76 & 158 & 80,20 & $0,59(0,26-1,32)$ & 0,185 \\
\hline Não & 39 & 19,80 & 45 & 88,24 & & \\
\hline $\begin{array}{l}\text { Problemas de saúde } \\
\text { referidos }\end{array}$ & & & & & & \\
\hline $0-3$ & 36 & 17,14 & 174 & 82,86 & $1,31(0,68-2,50)$ & 0,272 \\
\hline 4 ou mais & 09 & 22,50 & 31 & 77,50 & & \\
\hline \multicolumn{7}{|l|}{ Tipo de morbidade } \\
\hline \multicolumn{7}{|l|}{ Hipertensão Arterial } \\
\hline Sim & 30 & 19,35 & 125 & 80,65 & $1,28(0,64-2,52)$ & 0,477 \\
\hline Não & 15 & 15,79 & 80 & 84,21 & & \\
\hline \multicolumn{7}{|l|}{ Diabetes } \\
\hline Sim & 15 & 17,44 & 71 & 82,56 & $0,95(0,54-1,67)$ & 0,868 \\
\hline Não & 30 & 18,29 & 134 & 81,71 & & \\
\hline \multicolumn{7}{|l|}{ Osteoporose } \\
\hline Sim & 02 & 18,18 & 09 & 81,82 & $1,01(0,28-3,64)$ & 0,620 \\
\hline Não & 43 & 17,99 & 196 & 82,01 & & \\
\hline \multicolumn{7}{|l|}{ Artrite/artrose } \\
\hline Sim & 03 & 25,00 & 09 & 75,00 & $1,41(0,51-3,91)$ & 0,370 \\
\hline Não & 42 & 17,65 & 196 & 82,35 & & \\
\hline Cardiopatia & & & & & & \\
\hline Sim & 02 & 50,00 & 02 & 50,00 & $2,86(1,03-7,90)$ & 0,149 \\
\hline Não & 43 & 17,48 & 203 & 82,52 & & \\
\hline Problema de coluna & & & & & & \\
\hline Sim & 06 & 15,79 & 32 & 84,21 & $0,85(0,39-1,88)$ & 0,451 \\
\hline Não & 39 & 18,40 & 173 & 81,60 & & \\
\hline Incontinência urinaria & & & & & & \\
\hline Sim & 01 & 25,00 & 03 & 75,00 & $1,39(0,25-7,79)$ & 0,550 \\
\hline Não & 44 & 17,89 & 202 & 82,11 & & \\
\hline Uso de álcool & & & & & & \\
\hline $\operatorname{Sim}$ & 06 & 23,08 & 20 & 79,92 & $1,32(0,62-2,82)$ & 0,316 \\
\hline Não & 39 & 11,11 & 185 & 82,59 & & \\
\hline Uso do tabaco & & & & & & \\
\hline Sim & 06 & 18,75 & 26 & 81,25 & $1,04(0,48-2,27)$ & 0,535 \\
\hline Não & 39 & 17,89 & 179 & 82,11 & & \\
\hline Uso de medicamentos & & & & & & \\
\hline $\mathrm{Sim}$ & 39 & 21,31 & 144 & 78,69 & $0,34(0,13-0,92)$ & 0,012 \\
\hline Não & 04 & 7,41 & 50 & 92,59 & & \\
\hline Audição & & & & & & \\
\hline Sim & 41 & 18,30 & 21 & 84,00 & $1,14(0,44-2,92)$ & 0,515 \\
\hline Não & 04 & 16,00 & 183 & 81,70 & & \\
\hline Marcha & & & & & & \\
\hline Sim & 36 & 17,39 & 171 & 82,61 & $0,79(0,41-1,51)$ & 0,310 \\
\hline Não & 09 & 21,95 & 32 & 78,05 & & \\
\hline Equilíbrio & & & & & & \\
\hline Sim & 20 & 37,70 & 85 & 35,60 & $1,07(0,65-1,78)$ & 0,776 \\
\hline Não & 33 & 62,30 & 154 & 64,40 & & \\
\hline Atividade física & & & & & & \\
\hline Sim & 08 & 17,39 & 38 & 82,61 & $0,95(0,47-1,91)$ & 0,548 \\
\hline Não & 37 & 18,14 & 167 & 81,86 & & \\
\hline Familiares com depressão & & & & & & \\
\hline Sim & 23 & 41,82 & 32 & 58,18 & $3,70(2,24-6,12)$ & $<0,001$ \\
\hline Não & 22 & 11,28 & 173 & 88,72 & & \\
\hline Morte na família & & & & & & \\
\hline Sim & 25 & 23,81 & 80 & 76,19 & $1,72(1,01-2,93)$ & 0,031 \\
\hline Não & 20 & 13,79 & 125 & 86,21 & & \\
\hline AIVD & & & & & & \\
\hline Independente & 23 & 12,99 & 154 & 87,01 & $2,31(1,38-3,88)$ & 0,001 \\
\hline Dependente & 22 & 30,14 & 51 & 69,86 & & \\
\hline AVD & & & & & & \\
\hline Independente & 40 & 16,81 & 198 & 83,19 & $2,47(1,19-5,12)$ & 0,044 \\
\hline Dependente & 05 & 41,67 & 07 & 58,33 & & \\
\hline Autopercepção de saúde & & & & & & \\
\hline Ótima/ Boa & 30 & 20,83 & 114 & 79,17 & & \\
\hline Regular/péssima & 15 & 14,15 & 91 & 85,85 & $1,47(0,83-2,59)$ & 0,115 \\
\hline
\end{tabular}

RP: Razão de Prevalência; IC 95\%: Intervalo de Confiança para a proporção de 95\%; *teste de Fisher

Fonte: Dados da Pesquisa 


\section{DISCUSSÃO}

Este estudo mostrou que a prevalência de sintomas depressivos entre as idosas com 60 anos ou mais foi de $18,00 \%$. Comparado com pesquisas de base populacional conduzidas em diferentes países, observaram prevalências de sintomas depressivos que variaram entre $6,1 \%$ até $38,5 \%{ }^{16}$. Entre os mais suscetíveis à ocorrência de depressão estavam as mulheres com baixa renda, com história de eventos estressores na vida, regular percepção de vida e de saúde, com limitação funcional, doenças crônicas e comorbidades. Dessa forma, a depressão é frequentemente associada a maior risco de morbidades e de mortalidade, ocasionando o aumento na utilização dos serviços de saúde, à negligência no autocuidado, à adesão reduzida aos tratamentos terapêuticos e a maior risco de suicídio ${ }^{17}$.

A dependência para a realização das Atividades de Vida Diária (AVD) e Atividades Instrumentais de Vida Diária (AIVD) mostrou-se significativa para a prevalência de depressão em idosas na comunidade. Dessa forma, considerando que as escalas AVD e AIVD são imprescindíveis e requerem elaboração, coordenação e destreza, há mínima alteração dessas funções, como a perda da autonomia e consequente isolamento $\operatorname{social}^{17,18}$.

Ademais, além de desenvolver alguma deficiência e gerar sentimentos de ansiedade e insegurança, pode desencadear um episódio depressivo, ressaltando sobre a dependência AVD e AIVD, praticamente é uma causa de sintomas depressivos. No entanto, a depressão pode ser confundida com a doença orgânica de base, uma vez que o sofrimento e a incapacidade funcional favorecem o surgimento de sintomas como insônia, fadiga e perda da libido, mesmo quando não se estabelece o diagnóstico de depressão $^{18,19}$.

Apesar de não apresentar a associação de sintomas depressivos e problemas cardíacos, apresentou risco relativo de 2,86. Ademais, embora nos pareça que os problemas cardíacos e as incapacidades funcionais precedam os sintomas depressivos, esse provável fato deve ser avaliado com cautela, uma vez que o delineamento deste estudo está sujeito à causalidade reversa, ressaltando sobre o grau de dependência AVD e AIVD, a presença de incapacidades funcionais, praticamente, é uma causa de sintomas depressivos, estudos epidemiológicos de depressão e a investigação da associação com comorbidades cardiovasculares. ${ }^{17,20-22}$. Ademais, na pesquisa, a morte de algum membro da família demostrou associação com depressão nas idosas. Dessa forma, uma provável explicação torna-se comum surgir quadro depressivo no idoso, manifestando sintomas diversos, porém o mais comum é o desânimo e a perda da capacidade de amar, decorrentes do processo de culpa. No idoso em processo de luto podem ocorrer alguns distúrbios, como insônia, perda de apetite, falta de ar, aperto no peito, falta de energia, passividade, alucinações e ansiedade ${ }^{23,24}$.

$\mathrm{O}$ estudo afirma que entre os fatores de depressão em idosas está o uso de medicamentos. De acordo com estudo de Paradela, Lourenço e Veras ${ }^{23}$ os idosos com a presença de comorbidades e o uso de múltiplos fármacos fez com que diagnóstico e o tratamento da depressão se tornem mais complexos. Além disso, uma provável relação para a associação do uso de medicamentos com a depressão é que as idosas com algum grau de dependência, comprometem a autonomia para AVD e AIVD e isso inclui o uso de medicamentos ${ }^{24}$. 
Outro achado desta investigação foi a forte associação das idosas terem alguém na família com depressão, ou seja, estatisticamente elas têm probabilidade três vezes maior de desenvolver sintomas depressivos. Assim, quando há uma pessoa com depressão na família tudo se altera e os familiares mudam, construindo o papel de cuidador na interação quotidiana. As estratégias de cuidados desenvolvem-se de modo reativo em função do comportamento do doente, adquirindo contornos particulares de conteúdo nem sempre adequados à situação de saúde vivida ${ }^{25}$.

Assim, ter alguém com depressão em casa, exige que a família se adapte e se organize para enfrentar as atitudes daquela pessoa. Além disso, o suporte financeiro é importante, assim o cuidado com a idosa deve incluir uma boa escuta, observação, atenção e apoio, sempre que necessários, cabe à Unidade Básica de Saúde (UBS) compreender a funcionalidade da família da idosa que tem um membro com depressão, podendo subsidiar o planejamento de cuidados para responder a suas necessidades sociais e de saúde daquela família $^{24,26}$.

As idosas sofrem mais quando vivem em famílias desunidas em que há pouca expressão de afeto, compreensão e cumplicidade e, além disso, muita impaciência, raiva e agressividade, o empobrecimento das relações primárias se reflete na dinâmica cotidiana, o que torna o ambiente de convivência insuportável. O idoso se ressente quando filhos, netos, noras e genros não se entendem, pois, isso o priva dos encontros com familiares queridos e o isola ainda mais, outro ponto de tensão é a inexistência de manifestações de afetos entre os membros das famílias, a sensação de abandono dos familiares e amigos, e a falta de apoio para lidar com as situações depressivas $^{27,28}$. Dessa forma, para que a idosa possa usufruir de melhores condições de vida, deve haver carinho, respeito e cuidado no convívio familiar, o afeto, a ajuda mútua e a compreensão são fundamentais para uma boa qualidade de vida dessa idosa ${ }^{29,30}$.

\section{CONCLUSÃO}

A prevalência de sintomas depressivos entre as idosas foi de $18 \%$. A cardiopatia, o uso de medicamentos, dependência das AVD e AIVD, morte de familiares e alguém na família com depressão foram associados à depressão em idosas. Com esses resultados, vê-se a necessidade da detecção precoce dos sintomas depressivos, contribuindo para prevenir seus efeitos negativos na saúde e qualidade de vida dessas idosas.

Os profissionais de saúde da atenção básica devem estar aptos para uma prática dirigida não apenas para detectar sintomas de depressão, mas qualquer outra alteração que a idosa possa apresentar, entendendo que velhice não é sinônimo de doença, é importante a prática de consultas com uma avaliação multidimensional de modo abrangente e qualificado no atendimento à pessoa idosa.

Este estudo servirá para ajudar e difundir discussões sobre o referido tema, além de reafirmar a necessidade de mudanças na assistência à mulher idosa, enfatizando estratégias voltadas para a prevenção, intervenção em fatores de risco, diagnóstico precoce e tratamento da depressão. $\mathrm{O}$ estudo teve como limitação a condução da pesquisa por depender dos agentes de serviço de saúde, inviabilizando a pesquisa de forma eficaz, porém desperta o interesse pela realização de um estudo mais abrangente na região, envolvendo não só a população idosa e sim mulheres no geral pois há um alto índice de relatos na região de mulheres com depressão. 


\section{Referências}

1. Dawalibi NW, Anacleto GMC, Witter C, Goulart RMM, Aquino RC. Envelhecimento e qualidade de vida: análise da produção científica da Scielo. 2013; Estud. Psicol. (Campinas), 30(3): 393-403.

2. Magalhães JM, Carvalho AMB, Carvalho SM, Alencar DC, Moreira WC, Parente ACM. Depressão em idosos na estratégia saúde da família: uma contribuição para a atenção primária. REME - Rev Min Enferm. 2016; 20: e947.

3. Lima AMM, Silva HS, Galhardoni R. Envelhecimento bem-sucedido: trajetórias de um constructo e novas fronteiras. Interface (Botucatu). 2008; 12(27): 795-807.

4. Duncan BB, Chor D, Aquino EML, Bensenor IM, Mill JG, Schmidt MI et al . Doenças crônicas não transmissíveis no Brasil: prioridade para enfrentamento e investigação. Rev. Saúde Pública. 2012; 46(Suppl 1): 126-134.

5. Pinto DM, Jorge MSB, Pinto AGA, Vasconcelos MGF, Cavalcante CM, Flores AZT et al. Projeto terapêutico singular na produção do cuidado integral: uma construção coletiva. Texto contexto - enferm. 2011; 20(3): 493-502.

6. Cavalcante FG, Minayo MCS, Mangas RMN. Diferentes faces da depressão no suicídio em idosos. Ciênc. saúde coletiva. 2013; 18(10): 2985-2.

7. Silva RM, Mangas RMN, Figueiredo AEB, Vieira LJES, Sousa GS, Cavalcanti AMTS et al. Influências dos problemas e conflitos familiares nas ideações e tentativas de suicídio de pessoas idosas. Ciênc. saúde coletiva. 2015; 20(6): 1703-1710.

8. Gonçalves AMC, Teixeira MTB, Gama JRA, Lopes CS, Silva GA, Gamarra CJ et al . Prevalência de depressão e fatores associados em mulheres atendidas pela Estratégia de Saúde da Família. J. bras. psiquiatr. 2018; 67(2): 101-109.

9. Rocha EN, Lucena AF. Projeto Terapêutico Singular e Processo de Enfermagem em uma perspectiva de cuidado interdisciplinar. Rev. Gaúcha Enferm. 2018; 39: e2017-0057.

10. Gonçalves VC, Andrade KL. Prevalência de depressão em idosos atendidos em ambulatório de geriatria da região nordeste do Brasil (São Luís-MA). Rev. bras. geriatr. Gerontol. 2010; 13(2): 289-299.

11. Ferreira PCS, Tavares DMS. Prevalência e fatores associados ao indicativo de depressão entre idosos residentes na zona rural. Rev. esc. enferm. USP. 2013; 47(2): 401-407.

12. Lara HCAA. A prevalência de depressão em idosos assistidos nas Unidades de Saúde da Família em Várzea Grande, Mato Grosso. Trabalho de Conclusão de Curso, Centro Universitário de Várzea Grande, Cuiabá, Mato Grosso, 2018.

13. Katz S, Ford AB, Moskowitz RW, Jackson BA, Jaffe MW. Studies of illness in the aged. The Index of ADL: A Standardized Measure of Biological and Psychosocial Function. JAMA. 1963;185(12):914-9.

14. Lawton MP, Brody EM. Assessment of Older People: Self-Maintaining and Instrumental Activities of Daily Living. Gerontologist. 1969;9(3):179-86.

15. Yesavage JA, Sheikh JI. Geriatric depression scale (GDS): recent evidence and development of a shorter version. Clin Gerontol. 1986 Nov [cited 2017 May 01];5(1-2):165-73.

16. Hellwig N, Munhoz TN, Tomasi E. Sintomas depressivos em idosos: estudo transversal de base populacional. Ciênc. saúde coletiva. 2016; 21(11): 3575-3584.

17. Teston EF, Carreira L, Marcon SS. Sintomas depressivos em idosos: comparação entre residentes em condomínio específico para idoso e na comunidade. Rev. bras. enferm. 2014; 67(3): 450-456.

18. Brasil ISPS, Pondé MP. Sintomas ansiosos e depressivos e sua correlação com intensidade da dor em pacientes com neuropatia periférica. Rev. Psiquiatr. 2009; 31(1): 24-31.

19. Ashe S, Routray D. Prevalence, associated risk factors of depression and mental health needs among geriatric population of an urban slum, Cuttack, Odisha. J Geriatr Psychiatry. 2019; 34(12):1799-1807.

20. Teng CT, Humes EC, Demetrio FN. Depressão e comorbidades clínicas. Rev. psiquiatr. clín. [Internet]. 2005; 32(3): 149-159. 
21. Zhang Y, Chen Y, Ma L. Depression and cardiovascular disease in elderly: Current understanding. J Clin Neurosci. 2018; Jan; 47:1-5.

22. Oliveira JBA, Lopes RGC. O processo de luto no idoso pela morte de cônjuge e filho. Psicologia em Estudo. 2008; 13(2), 217-221.

23. Paradela EMP, Lourenço RA, Veras RP. Validação da escala de depressão geriátrica em um ambulatório geral. Rev. Saúde Pública. 2005; 39(6): 918-923.

24. Souza RA, Costa GD, Yamashita CH, Amendola F, Gaspar JC, Alvarenga MRM et al. Funcionalidade familiar de idosos com sintomas depressivos. Rev. esc. enferm. USP. 2014; 48(3): 469-476.

25. Marques MF, Lopes MJ. O cuidador familiar no olhar da pessoa com depressão. Revista Portuguesa de Enfermagem de Saúde Mental. 2015; (spe2): 51-56.

26. Gullich I, Duro SMS, Cesar JA. Depressão entre idosos: um estudo de base populacional no Sul do Brasil. Rev. bras. epidemiol. 2016; 19(4): 691-701.

27. Maciel ACC, Guerra RO. Prevalência e fatores associados à sintomatologia depressiva em idosos residentes no Nordeste do Brasil. J. bras. psiquiatr. 2006; 55( 1 ): 26-33. A

28. Stopa SR, Malta DC, Oliveira MM, Lopes CS, Menezes PR, Kinoshita RT. Prevalência do autorrelato de depressão no Brasil: resultados da Pesquisa Nacional de Saúde, 2013. Rev. bras. epidemiol. 2015; 18(Suppl 2): 170-180.

29. Bretanha AF, Facchini LA, Nunes BP, Munhoz TN, Tomasi E, Thumé E. Sintomas depressivos em idosos residentes em áreas de abrangência das Unidades Básicas de Saúde da zona urbana de Bagé, RS. Rev. bras. epidemiol. 2015; 18(1): 1-12.

30. Campos ACV, Almeida MHM, Campos GV, Bagutchi TF. Prevalence of functional incapacity by gender in elderly people in Brazil: a systematic review with metaanalysis. Rev bras geriatr gerontol. 2016 May/June;19(3):545-59.

\section{Como citar este artigo:}

de Lara HCAA, Melo CA, Silva EC, Silva IA, Oliveira JS, Santana FS. Prevalência de depressão em mulheres idosas assistidas na atenção básica. Rev. Aten. Saúde. 2020; 18(64): $42-51$. 